\title{
A Multiple-Valued Logic and Memory With Combined Single-Electron and Metal-Oxide-Semiconductor Transistors
}

\author{
Hiroshi Inokawa, Member, IEEE, Akira Fujiwara, Member, IEEE, and Yasuo Takahashi, Member, IEEE
}

\begin{abstract}
Devices that combine single-electron and metaloxide-semiconductor (MOS) transistors are newly proposed as basic components of multiple-valued (MV) logic, such as a universal literal gate and a quantizer. We verified their operation using single-electron and MOS transistors fabricated on the same wafer by pattern-dependent oxidation of silicon. We also discuss their application to an analog-to-digital converter, a MV adder, and MV static random-access memory.
\end{abstract}

Index Terms-Literal gate, MOSFET, multiple-valued logic, multiple-valued memory, quantizer, single-electron transistor (SET).

\section{INTRODUCTION}

A DVANCES in large-scale integrated circuits (LSIs) have been based mostly on binary logic, and some research on emerging single-electron devices (SEDs) has followed the same path [1]-[5]. However, the problems in present LSI technology, such as increased interconnect delay and power consumption [6], [7], cannot be solved by simply replacing conventional devices with SEDs, or it may even get worse due to the low drivability originating from the high tunneling resistance and the high off-state leakage related to the low charging energy in the present technology [2], [3]. An advanced logic scheme that achieves higher functionality with fewer components and interconnections, is thus very desirable for SEDs. Multiple-valued (MV) logic is recognized as an advanced logic scheme in this sense [8]-[12], but its implementation has been limited to conventional devices, which are inherently singlethreshold or single-peak and thus not fully suited for MV logic. In contrast, SEDs are very suitable for MV logic because the discreteness of the electronic charge in the Coulomb island can be directly related to multiple-valued operation. Actually, some single-electron MV logics have been proposed [13], [14], but the MV operation has not yet been verified experimentally mainly because the schemes require unrealistic accuracy or limitations with respect to device parameters and operating conditions.

For the same reason, SEDs are also very suitable for MV memory. Unlike MV logics, MV schemes are already practically used in MOS read-only [12] and flash memories [15], [16]. Floating-node-type memories with SEDs have also been demonstrated experimentally [17], [18]. However, static memory, which features a fast simple write operation and

\footnotetext{
Manuscript received June 10, 2002; revised November 4, 2002. The review of this paper was arranged by Editor S. Datta.

The authors are with NTT Basic Research Laboratories, NTT Corporation, Kanagawa 243-0198 Japan (e-mail: inokawa@aecl.ntt.co.jp).

Digital Object Identifier 10.1109/TED.2002.80842
}

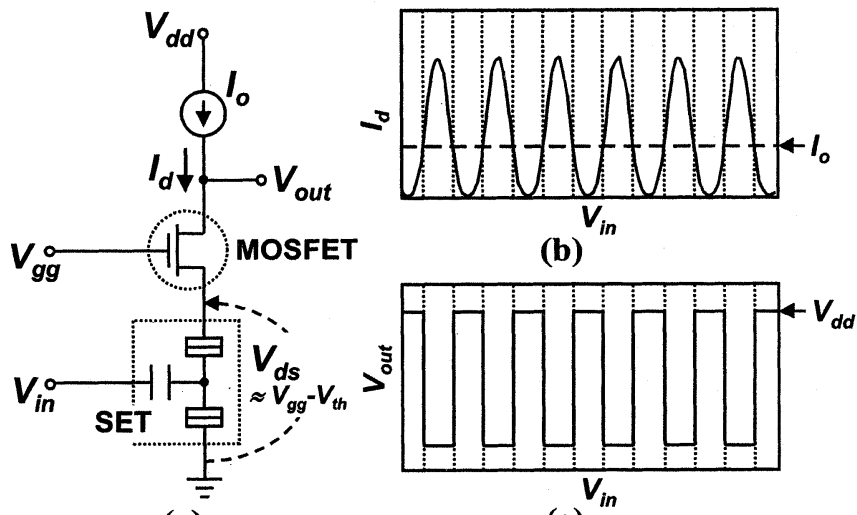

(a)

Fig. 1. (a) Schematic of the universal literal gate comprising a SET, a MOSFET and a constant-current (CC) load $I_{o}$. (b) $\boldsymbol{I}_{d}-V_{\text {in }}$ characteristics, which are almost completely independent of $V_{\text {out }}$ since the $V_{d s}$ of the SET is kept nearly constant at $V_{g g}$ minus $V_{t h}$, the threshold voltage of the MOSFET. (c) Expected transfer $\left(V_{i n}-V_{o u t}\right)$ characteristics.

stable retention while the power is on, has not practically been achieved with SEDs. A bistable flip-flop (inverter string) has been proposed [2], but it would be difficult to develop it into a MV memory. Preliminary results on a MV memory have been reported recently by us [19], but they were only for a slow and complex current sweep measurement.

In this paper, we introduce a series of single-electron MV logic and memory, in which MOSFETs supplement single-electron transistors (SETs) to attain practical characteristics. We demonstrate the operation of basic components of MV logic, such as a universal literal gate and a quantizer, which is equivalent to a MV static memory, using devices fabricated by the Si-based pattern-dependent oxidation (PADOX) process [20], [21]. The usefulness of the proposed single-electron MV logic and memory is discussed using an analog-to-digital converter (ADC), a MV adder, and MV static random-access memory (SRAM) as examples.

\section{OPERATION PRINCIPLE}

A universal literal gate is a basic component of the MV logic. Since it converts a MV input to a periodic binary output, a SET, which has periodic $\boldsymbol{I}_{d}-\boldsymbol{V}_{g s}$ characteristics, is readily applicable. However, a SET has disadvantages as a circuit element. The drain current heavily depends on the drain voltage, and thus the output resistance is low. The maximum voltage gain of a simple inverter, $\boldsymbol{C}_{g} / \boldsymbol{C}_{d}$, where $\boldsymbol{C}_{g}$ is the gate capacitance and $C_{d}$ is the drain capacitance, is very small, usually less than one 


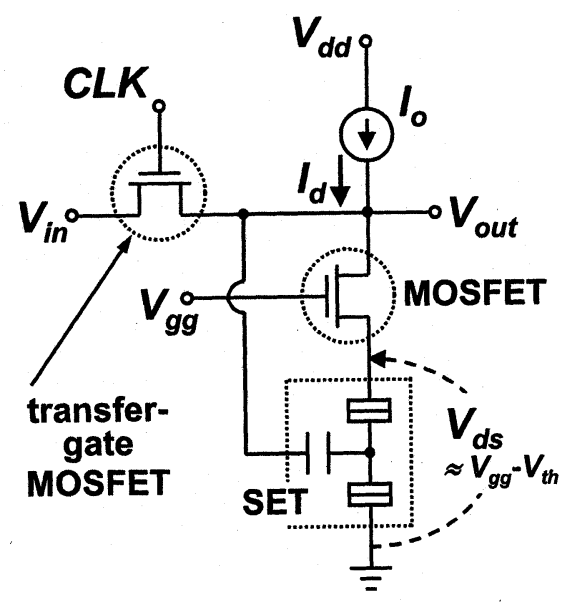

(a)

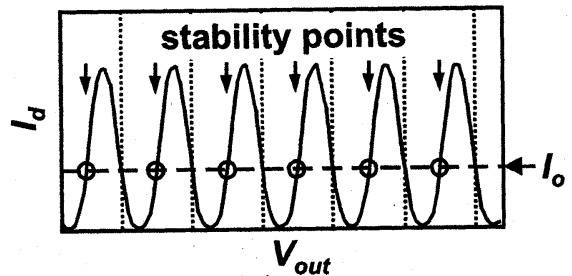

(b)

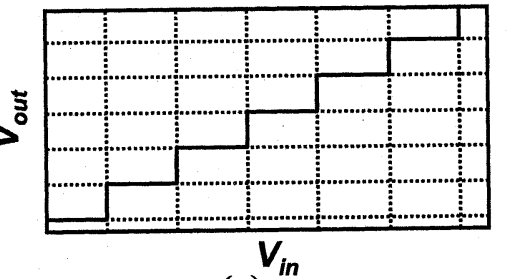

(c)

Fig. 2. (a) Schematic of the proposed quantizer (static memory). (b) Two-terminal $\boldsymbol{I}_{d}-\boldsymbol{V}_{\text {out }}$ characteristics of the SET-MOSFET device with the SET gate shorted to the MOSFET drain. (c) Expected transfer $\left(\boldsymbol{V}_{\text {in }}-\boldsymbol{V}_{\text {out }}\right)$ characteristics. $\boldsymbol{V}_{\text {in }}$ is transferred to $V_{\text {out }}$ through the transfer gate MOSFET and is quantized to a stability point after the gate is cut off.

or a little more than one. Applicable drain voltage is also limited to a small value, $\boldsymbol{e} / \boldsymbol{C}_{\Sigma}$, where $\boldsymbol{e}$ is an electronic charge and $\boldsymbol{C}_{\Sigma}$ is the total capacitance around the Coulomb island, to sustain the Coulomb-blockade condition. In order to overcome these disadvantages and to make a practical MV logic, we constructed a hybrid circuit containing MOSFETs. Fig. 1(a) is a schematic of the universal literal gate comprising a SET, a MOSFET and a constant-current (CC) load $\boldsymbol{I}_{o}$. A MOSFET with a fixed gate bias of $V_{g g}$ is used here to keep the SET drain voltage almost constant at $V_{g g}-V_{t h}$, where $V_{t h}$ is the MOSFET threshold voltage. This $\boldsymbol{V}_{g g}-\boldsymbol{V}_{t h}$ is set low enough to sustain the Coulomb-blockade condition. Current through this circuit increases and decreases periodically as a function of input voltage [Fig. 1(b)] unless the CC load is connected. The current is determined only by the input voltage; it is independent of the output voltage, because the drain voltage of the SET is kept constant by the MOSFET. When the CC load is connected and the increasing drain current crosses the load line of $\boldsymbol{I}_{o}$, the output voltage switches very sharply from high to low. Then, at the second crossing point, it switches from low to high, and so on. Thus, sharp square-wave-like input-output characteristics with a large voltage swing are obtained [Fig. 1(c)]. Note that the CC load can be realized experimentally by using a current-mode output from a semiconductor parameter analyzer with compliance (voltage limit) of $\boldsymbol{V}_{d d}$. Practically, a depletion-mode MOSFET with its gate and source shorted can be utilized as will be shown later.

Fig. 2(a) is a schematic of the proposed quantizer. Since $I_{d}$ is independent of $\boldsymbol{V}_{\text {out }}$, the periodic $\boldsymbol{I}_{d}-\boldsymbol{V}_{g s}$ characteristics of a SET can be converted to two-terminal $\boldsymbol{I}_{d}-V_{\text {out }}$ characteristics by simply connecting the SET gate to the MOSFET drain [Fig. 2(b)]. With a CC load $\boldsymbol{I}_{o}$, many stability points appear, and each stability point has a pull-in range separated by the dashed lines. $\boldsymbol{V}_{\text {in }}$ fed through the transfer-gate MOSFET is quantized to a stability point after the gate is cut off. This results in the staircase-like $\boldsymbol{V}_{\text {in }}-\boldsymbol{V}_{\text {out }}$ characteristics shown in Fig. 2(c). This quantizer can be regarded as a static memory as it has a latching capability, i.e., the stable condition is kept even after the input is removed as long as the power is on. The

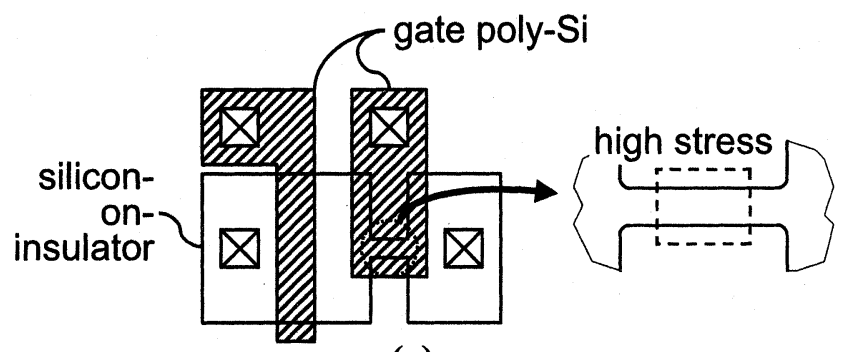

(a)

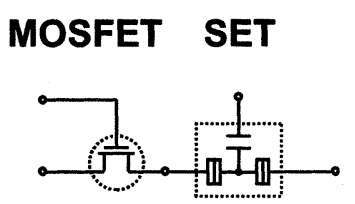

(b)

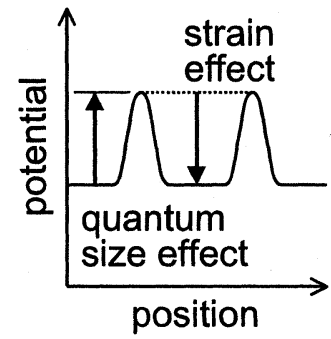

(c)

Fig. 3. (a) Possible layout of the integrated SET and MOSFET. (b) Circuit diagram corresponding to the layout above. (c) Potential profile along the length of the narrow wire, where a SET is created by pattern-dependent oxidation (PADOX) [20], [21]. Since areas outside the wire can readily be used for MOSFETs, PADOX is highly compatible with a CMOS process.

difference between it and our previous MV memory [19] is the addition of the transfer-gate MOSFET. We can directly select any stability points in a short time through this MOSFET.

Note that the number of periods in the universal literal characteristics [Fig. 1(c)] or voltage levels in the quantizer characteristics [Fig. 2(c)] is infinite in principle. This is a unique feature of our SET-based MV logic, and leads to a considerable reduction of the number of devices in a circuit.

\section{EXPERIMENTS}

The layout of the integrated SET and MOSFET, and the corresponding circuit diagram are shown in Fig. 3(a) and (b). Both the 


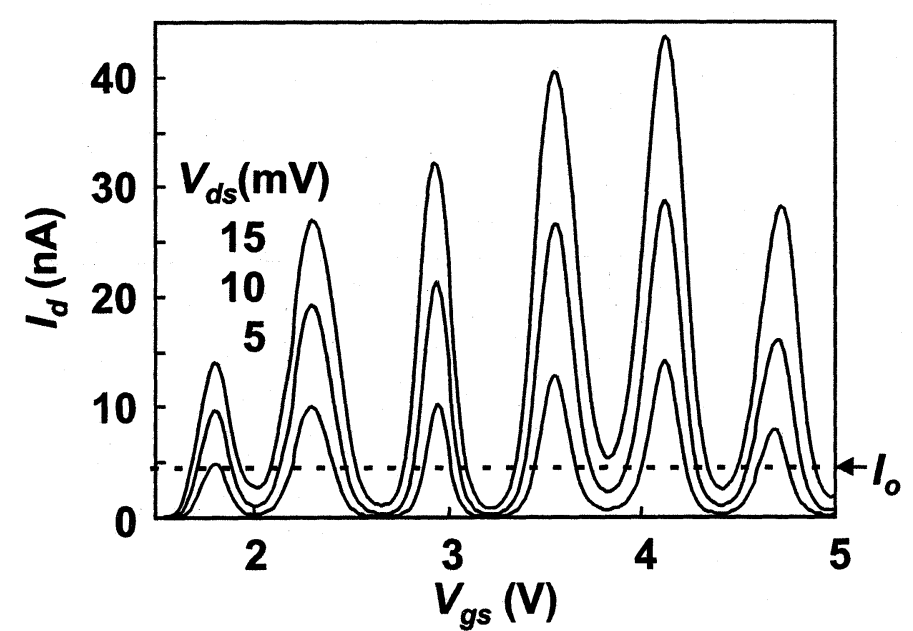

Fig. 4. $I_{d}-V_{g s}$ characteristics of a SET fabricated by the PADOX process, measured at $27 \mathrm{~K}$.

SET and the MOSFET were fabricated on a thin silicon-on-insulator (SOI) layer whose original thickness before oxidation was $30 \mathrm{~nm}$. The SET was created in a narrow wire region by PADOX [20], [21] at $1000^{\circ} \mathrm{C}$ in pure oxygen ambient. The original length and width of the wire were both $50 \mathrm{~nm}$, and the latter shrank to about $10 \mathrm{~nm}$ after oxidation. The quantum size effect raises the potential in the wire, but in the middle of the wire the high compressive stress generated by the oxidation reduces the bandgap [Fig. 3(c)] [21]. This creates two tunnel barriers and an island sandwiched between them, which constitute a SET. Since the areas outside the wire can easily be used for MOSFETs, PADOX is highly compatible with the CMOS process.

Fig. 4 shows the $\boldsymbol{I}_{d}-\boldsymbol{V}_{g s}$ characteristics of a SET with drain voltage as a parameter. Periodic drain-current peaks are clearly seen along with a strong drain voltage dependence of current. As is often observed in semiconductor SETs, the peak heights are uneven and the positions are not perfectly periodic, but the degree of irregularity is acceptable for the following demonstrations of 6-valued operation.

Fig. 5 shows the Coulomb diamond plot for the device shown in Fig. 4. From this figure, gate capacitance $\boldsymbol{C}_{g}$, source capacitance $\boldsymbol{C}_{s}$, drain capacitance $\boldsymbol{C}_{d}$, and tunnel resistance were calculated to be $0.27 \mathrm{aF}, 2.7 \mathrm{aF}, 2.7 \mathrm{aF}$, and $80 \sim 220 \mathrm{k} \Omega$, respectively. The maximum voltage gain $\boldsymbol{C}_{g} / \boldsymbol{C}_{d}$ (inverting) and applicable voltage $\boldsymbol{e} / \boldsymbol{C}_{\Sigma}$ for this SET are as low as 0.1 and $28 \mathrm{mV}$, respectively. However, there is no problem in constructing the proposed MV logic and memory, because these values are supplemented by the MOSFET.

Fig. 6 shows the subthreshold characteristics of a MOSFET fabricated on the same SOI wafer for drain voltages of $5 \mathrm{~V}$ and $10 \mathrm{mV}$. The gate length and width and the gate oxide thickness are $14 \mu \mathrm{m}, 12 \mu \mathrm{m}$, and $90 \mathrm{~nm}$, respectively. In order to maintain the constant drain voltage of the SET, the subthreshold slope of the MOSFET should be steep and the $V_{t h}$ shift due to the drain voltage should also be small. The actual subthreshold slopes are 95 and $62 \mathrm{mV} /$ decade for drain voltages of $10 \mathrm{mV}$ and $5 \mathrm{~V}$, respectively, and the $\boldsymbol{V}_{t h}$ shift is $53 \mathrm{mV}$ at the drain current of operation (4.5 nA). Despite these rather large values [22], the qualitative behavior of the proposed literal gate and the quan-

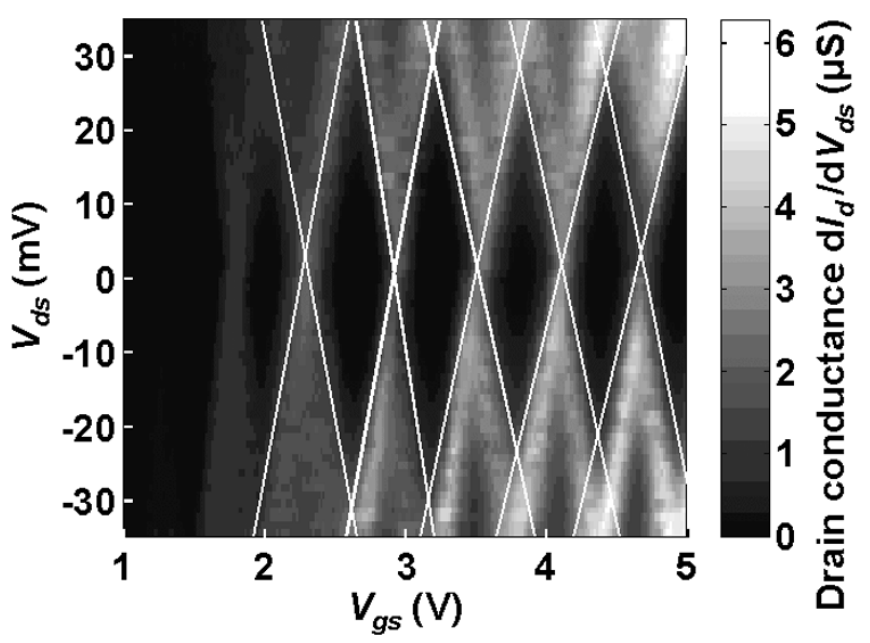

Fig. 5. Coulomb diamond plot of the SET shown in Fig. 4. From this figure, gate capacitance $\boldsymbol{C}_{g}$, source capacitance $\boldsymbol{C}_{s}$, drain capacitance $\boldsymbol{C}_{d}$ and tunneling resistance are calculated to be $0.27 \mathrm{aF}, 2.7 \mathrm{aF}, 2.7 \mathrm{aF}$ and $80 \sim 220 \mathrm{k} \Omega$, respectively.

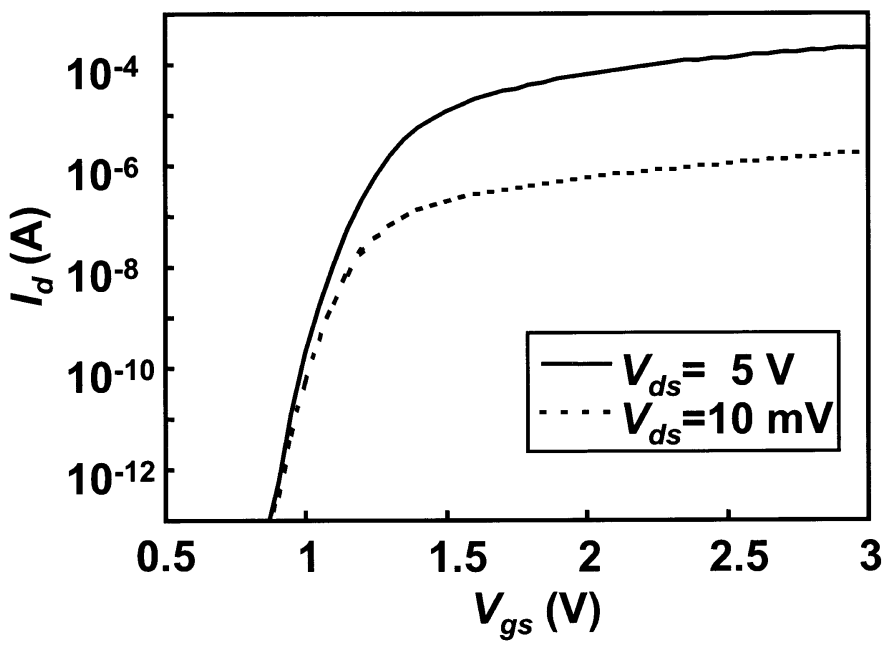

Fig. 6. $I_{d}-\boldsymbol{V}_{g s}$ characteristics of a MOSFET fabricated on the same SOI wafer as the above SET, measured at $27 \mathrm{~K}$. Effective channel width and length and gate oxide thickness are $12 \mu \mathrm{m}, 14 \mu \mathrm{m}$ and $90 \mathrm{~nm}$, respectively. Threshold voltage $V_{t h}$, corresponding to $I_{d}=4.5 \mathrm{nA}$ and $\boldsymbol{V}_{d s}=3 \mathrm{~V}$, is $1.07 \mathrm{~V}$, and transconductance $\boldsymbol{G}_{m}$ at $\boldsymbol{V}_{d s}=\boldsymbol{V}_{g s}=3 \mathrm{~V}$ is $151 \mu \mathrm{S}$.

tizer can be observed. However, the peak-to-valley current ratios (PVCRs) of $\boldsymbol{I}_{d}$ in Figs. 1(b) and 2(b) become suppressed, and the ranges of $\boldsymbol{V}_{\text {out }}$ in Figs. 1(c) and 2(c) become narrower.

Fig. 7 shows the input-output characteristics of the universal literal gate. The output voltage increases and decreases periodically with the input voltage, reflecting the original characteristics of the SET. The output shows a clear saturation level of as high as $5 \mathrm{~V}$, which is sufficient to drive the next stages of SET or MOSFET circuits. The voltage gain, which represents the sharpness of the transition, is the product of the maximum available voltage gains of the SET and the MOSFET, according to the analysis of a cascode amplifier [23]. If the $\boldsymbol{I}_{d}-\boldsymbol{V}_{d s}$ characteristics of the MOSFET were perfectly flat in the saturation region, the voltage gain would be infinite and the abrupt transition shown in Fig. 1(c) could be obtained. In reality, the voltage gain of the present MOSFET is only 400, and the resultant slope of the transition is about 40. Though this slope is much larger than the gain of the discrete SET, it still needs further improve- 


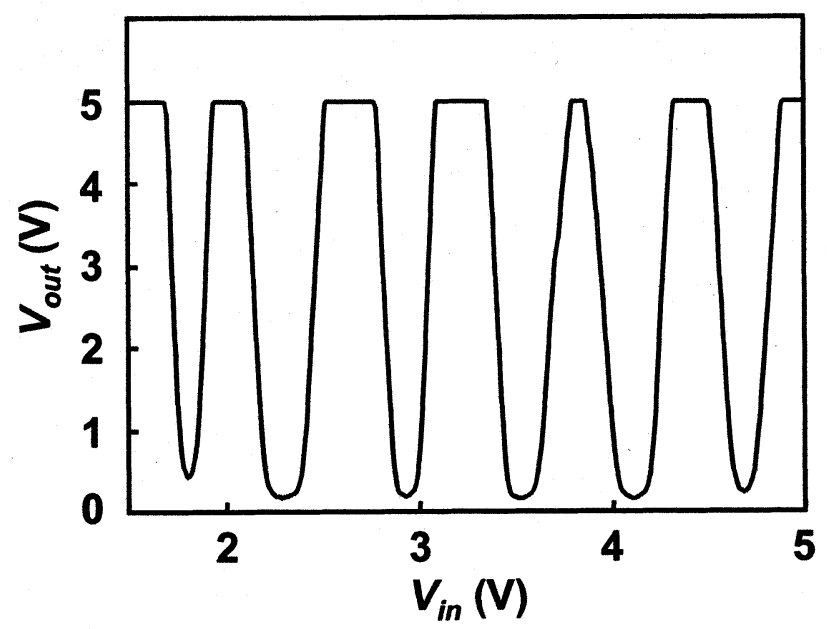

Fig. 7. Measured transfer $\left(\boldsymbol{V}_{\text {in }}-V_{\text {out }}\right)$ characteristics of the proposed literal gate [Fig. 1(a)] comprising a SET, a MOSFET and a CC load. The $\boldsymbol{V}_{g g}$ is set to $1.08 \mathrm{~V}$ to attain a SET drain voltage of about $10 \mathrm{mV}$. The CC load is realized by a current-mode output of $4.5 \mathrm{nA}$ from a semiconductor parameter analyzer with compliance (voltage limit) of $5 \mathrm{~V}$. Originally, the SET $I_{d}-V_{g s}$ characteristics have a large $V_{d s}$ dependence as indicated in Fig. 4, and this $\boldsymbol{V}_{d s}$ dependence is alleviated by the MOSFET, resulting in a large voltage swing and sharp rises and falls.

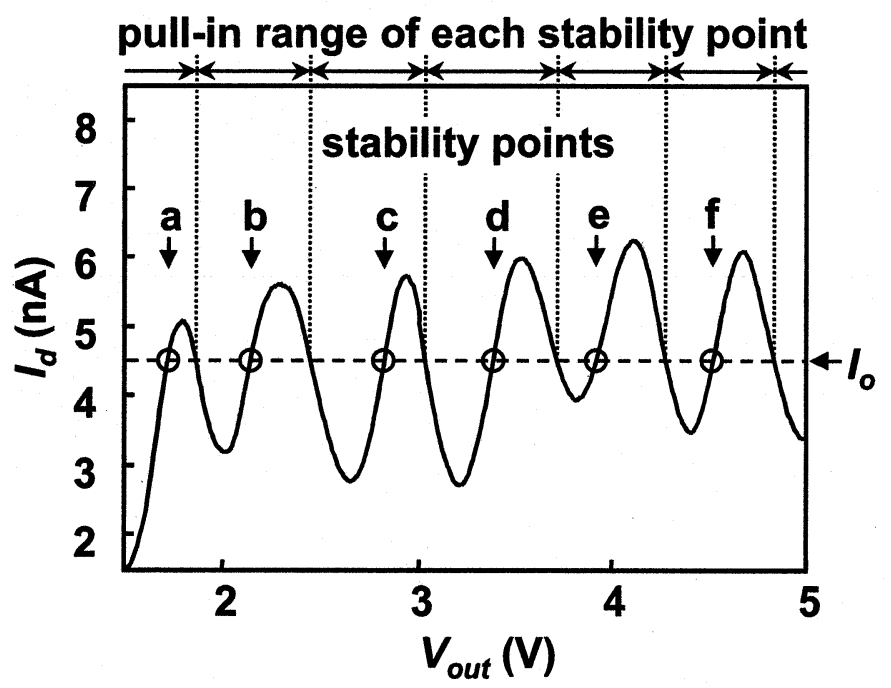

Fig. 8. Two-terminal $I_{d}-V_{\text {out }}$ characteristics of the SET-MOSFET device with the SET gate shorted to the MOSFET drain, measured at $1.08 \mathrm{~V}$ of $V_{g g}$ [19]. Stability points $(\mathbf{a} \sim \mathbf{f})$ and the corresponding pull-in ranges expected for the current load of $4.5 \mathrm{nA}$ are also shown.

ment, that could be attained by increasing the output resistance and/or the transconductance of the MOSFET.

Fig. 8 shows the two-terminal $\boldsymbol{I}_{d}-\boldsymbol{V}_{\text {out }}$ characteristics of the SET-MOSFET device with the SET gate shorted to the MOSFET drain [19]. The current increases and decreases periodically, reflecting the $\boldsymbol{I}_{d}-\boldsymbol{V}_{g s}$ characteristics of the discrete SET. If we were to connect a CC load of $4.5 \mathrm{nA}$, stability points $\mathbf{a} \sim \mathbf{f}$ corresponding to quantized levels would appear, and each point would have a pull-in range nearly equal to the voltage range of the quantizer input associated with the quantized level. The PVCR of the SET-MOSFET device shown in Fig. 8 is 2.1 at most and much smaller than that of the discrete SET in Fig. 4. This is because the MOSFET is not an ideal constant voltage source to the SET drain; that is, increased drain voltage increases the valley current and decreased drain voltage decreases the peak current. The PVCR can easily be improved to 28 for instance by simply decreasing the $\boldsymbol{C}_{\Sigma}$ by a factor of 5 and increasing the tunneling resistance by the same amount [19].

Quantizer operation was verified using the setup shown in Fig. 9. The central SET and MOSFET were on the same wafer, but the transfer-gate MOSFET1 and the MOSFET2 for probing were connected externally. A triangular wave was fed to $V_{i n}$, and the gate of MOSFET1 was driven by short pulses of $\boldsymbol{C L} \boldsymbol{K}$. Different voltage levels in the triangular wave were sampled by the MOSFET1, transferred to the storage node, $\boldsymbol{V}_{\text {out }}$, and quantized. The waveforms are shown in Fig. 10. $V_{\text {out }}$ was quantized to levels $\mathbf{a} \sim \mathbf{f}$ that correspond to stability points in Fig. 8 . The operating speed in the figure is not limited by the intrinsic performance of the device, but by the large stray capacitance of $370 \mathrm{pF}$ at $\boldsymbol{V}_{\text {out }}$.

\section{DISCUSSION}

\section{A. Application to ADC and MV Adder}

Fig. 11 is a block diagram of a 3-bit flash ADC based on the combined SET-MOSFET scheme. Input is quantized by the proposed circuit, and then capacitively divided into the literal gate corresponding to each output digit. Although a conventional $\boldsymbol{n}$-bit flash ADC requires $\boldsymbol{n}^{2}-1$ components, such as comparators, the SET ADC [24], [25] requires only $\boldsymbol{n}$ components. This particular ADC with the quantizer at the front end and the SET-MOSFET literal gates does not require comparators, latches, or ramp generators at each literal gate because the quantizer has a latching capability and the literal gates have squarewave-like transfer characteristics with saturated high and low levels. The use of Gray code is not necessary either, since the quantizer prevents the entry of intermediate voltage levels.

Fig. 12 is a circuit diagram of the ADC in Fig. 11. Depletion-mode MOSFETs are used in place of CC loads (M3, M5, M7 and M9) and to eliminate the gate bias voltage for the stacked MOSFETs (M2, M4, M6 and M8). The MOSFETs for CC loads operate in the saturation region, and the $\boldsymbol{V}_{t h}$ or channel width or length must be adjusted to attain the target current $\boldsymbol{I}_{o}$. The absolute $V_{t h}$ of the stacked MOSFETs, which corresponds to the drain voltage of the SETs, should be low enough to sustain the Coulomb blockade condition.

The circuit shown in Fig. 12 is remarkably simple in that a literal gate is constructed with only three elements and a quantizer with only four. In the conventional implementation with MOSFETs, the main blocks of the ADC, excluding the quantizer, require 28 transistors [8]. The proposed circuit requires only half, i.e., nine transistors and five capacitors.

Fig. 13 shows the block diagram of a full adder for redundant positive-digit number representation PD2-3 [8]. The main parts of this adder are the same as those of the 3-bit ADC in Figs. 11 and 12 , and $\oplus \mathrm{s}$ are the linear sums. This number system is 4 -valued, and each digit can take $0,1,2$, or 3 . For example, decimal number 19 can be expressed by $(3,2,3)_{P D 2-3}$, and 18 can be expressed by $(3,2,2)_{P D 2-3}$. It can be seen that the sum of these numbers is calculated by this circuit to be $(1,2,1,0,1)_{P D 2-3}$, which corresponds to the decimal 37 and is the right answer. As can be expected from the results for the 3-bit ADC, the number of elements 


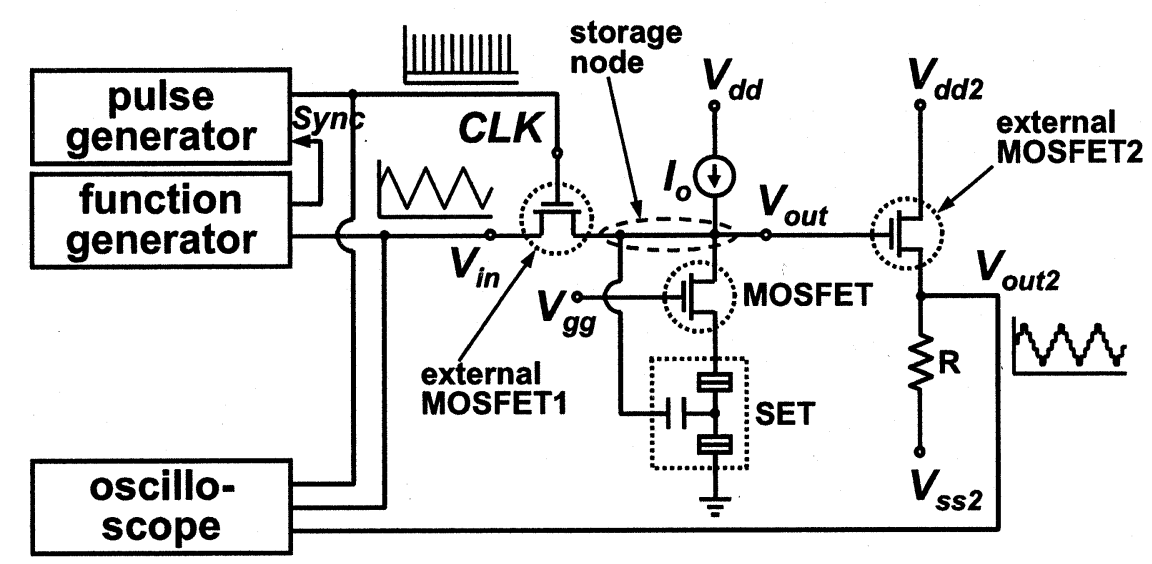

Fig. 9. Measurement setup for the quantizer proposed in Fig. 2(a). The central SET and MOSFET are on the same wafer. The external MOSFET1 is a transfer gate, and MOSFET2 is used as an FET probe to measure the $V_{\text {out }}$ sustained by a small current $(\sim \mathrm{nA})$.

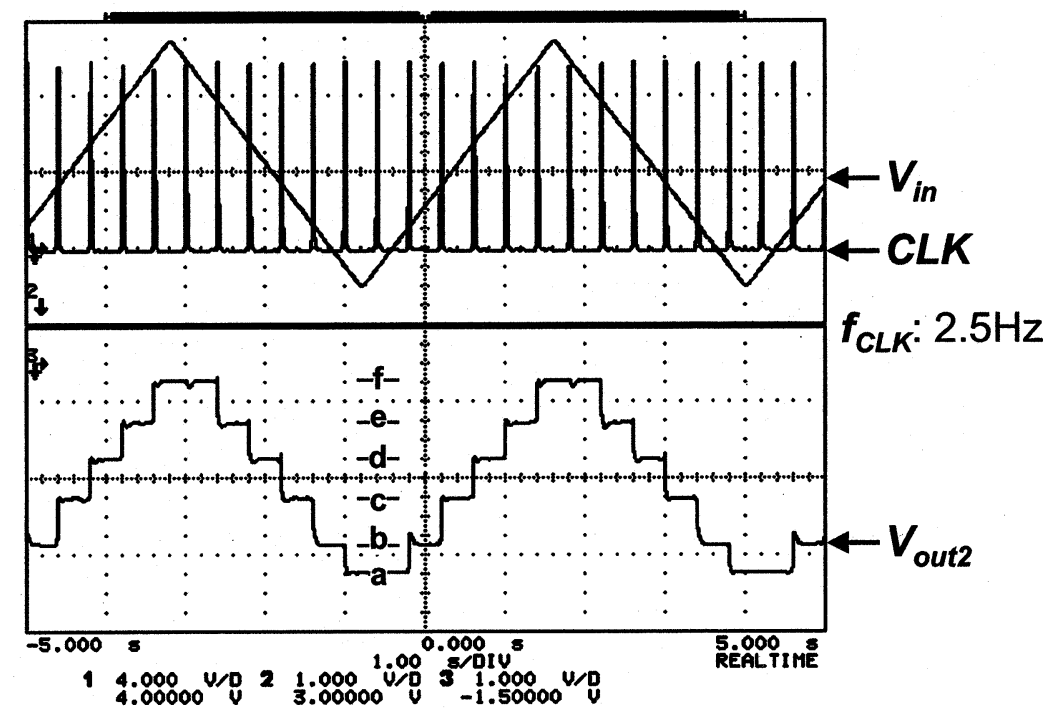

Fig. 10. Quantizer operation measured by the setup in Fig. 9, with $V_{g g}$ of $1.08 \mathrm{~V}$ and a CC load of $4.5 \mathrm{nA}$. Operation speed is not limited by the intrinsic performance of the device, but by the large stray capacitance of $370 \mathrm{pF}$ at $V_{\text {out }}$.

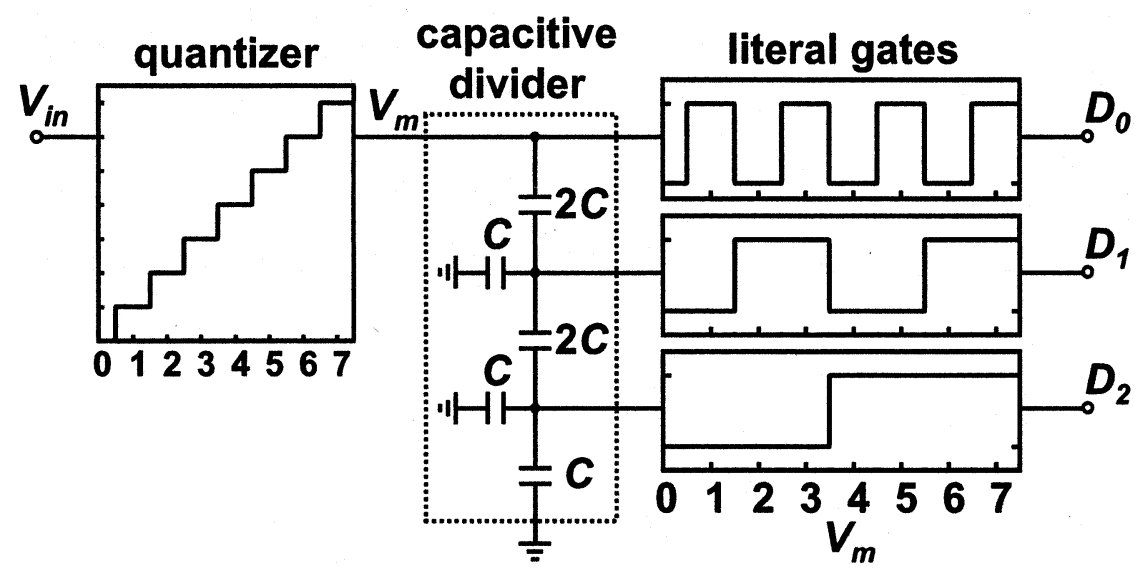

Fig. 11. A block diagram of a 3-bit flash ADC. A conventional $\boldsymbol{n}$-bit flash ADC requires $\boldsymbol{n}^{2}-1$ components, whereas the SET ADC [24], [25] requires only $\boldsymbol{n}$. This particular ADC with the quantizer at the front end and with the SET-MOSFET literal gates does not require comparators, latches, or ramp generators at each literal gate. Gray code is not necessary either.

in this adder can be reduced by half through the use of the combined SET-MOSFET scheme. In addition, this multiple-valued arithmetic itself has the merit of high-speed operation due to the elimination of carry propagation. 


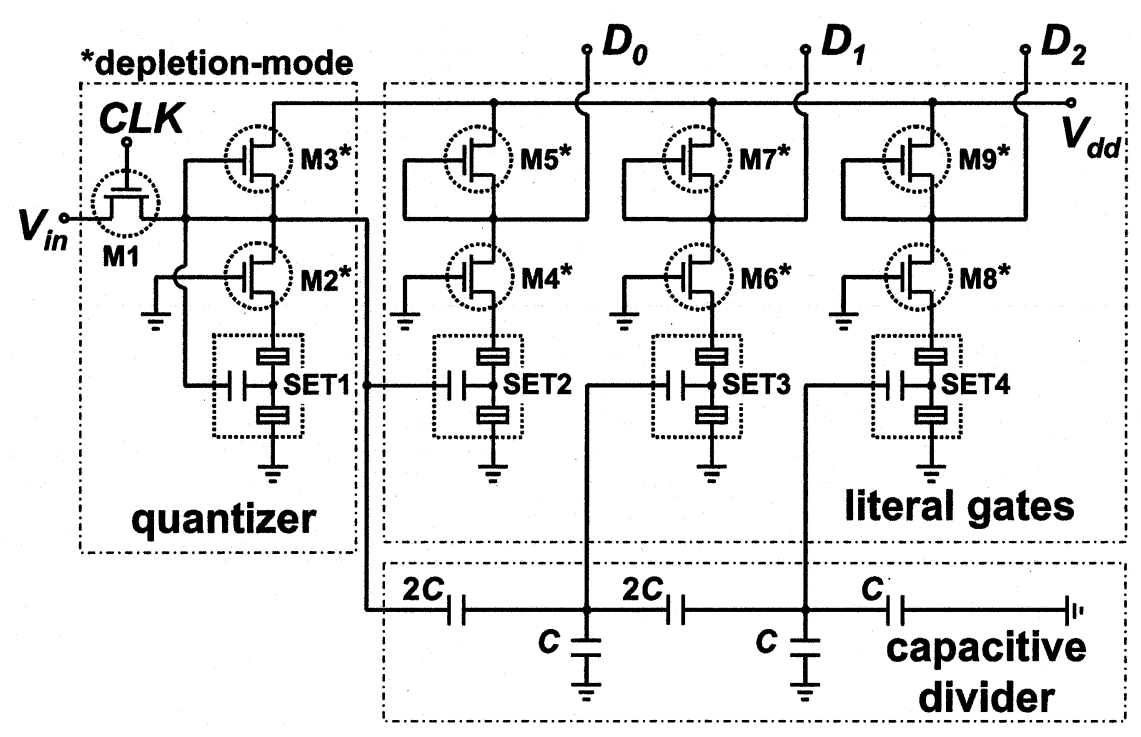

Fig. 12. The circuit diagram of the 3-bit ADC in Fig. 11. Depletion-mode MOSFETs, M2, M4, M6 and M8, keep the drain voltage of the SETs nearly constant at the absolute threshold voltage. Other depletion-mode MOSFETs, M3, M5, M7 and M9, serve as CC loads.

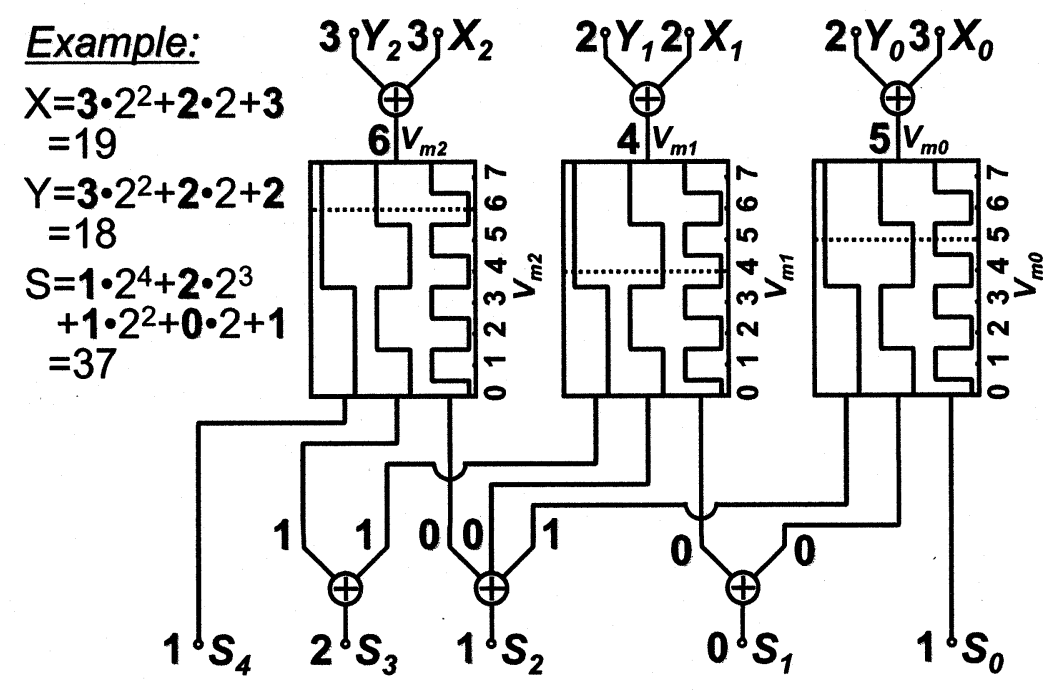

Fig. 13. A full adder for redundant positive-digit number representation PD2-3 [8] consisting of three ADC blocks without a quantizer, and linear sums ( $\oplus$ ). This adder is free from carry propagation, and is thus fast. By using the SET-MOSFET devices, multiple-valued logics of this kind can be made with half the number of elements in conventional implementation.

\section{B. Application to SRAM}

Fig. 14(a) shows a four-transistor memory cell for large-scale SRAMs. Again, a depletion-mode grounded-gate MOSFET M1 is used to sustain the SET drain voltage, and another depletion-mode MOSFET, M2, with its gate and source shorted serves as a CC load. The pass-transistor M3 controls the access to the cell. In write operation, the voltage applied to the bit line (BL) is transferred through $\mathbf{M 3}$, and is quantized to a stability point after M3 is cut off. In the read operation, the stored voltage level is transferred back to BL through $\mathbf{M 3}$, and then sensed by peripheral circuits. Fig. 14(b) shows an example of the cell layout. The cell has an area of $31.1 \boldsymbol{F}^{2}$, where $\boldsymbol{F}$ is half the minimum wiring pitch. Thus, this cell not only has a multiple-valued capability, but is much smaller than a CMOS binary SRAM cell.

\section{Operating Speed}

The current that can be supplied from the proposed logic is determined by the margin between the load current $I_{o}$ and the peak/valley currents, and is of the order of $1 \mathrm{nA}$ (Fig. 8). If we assume the voltage swing of $1 \mathrm{~V}$ and the load capacitance of $100 \mathrm{fF}$, which roughly corresponds to the wiring length of $1 \mathrm{~mm}$, the delay time becomes as large as $100 \mu$ s. Since the input capacitance of a SET is very small, the load capacitance can probably be reduced to $100 \mathrm{aF}$ by limiting fan-out and reducing wiring length. The $100 \mathrm{aF}$ is still much larger than the total capacitance $\boldsymbol{C}_{\Sigma}$ around the Coulomb island, which satisfies the requirement for stable SET operation. The available output current can be increased to over $10 \mathrm{nA}$ by improving the peak-to-valley ratio and adjusting the SET drain voltage. In addition, the voltage swing can be reduced by nearly one 


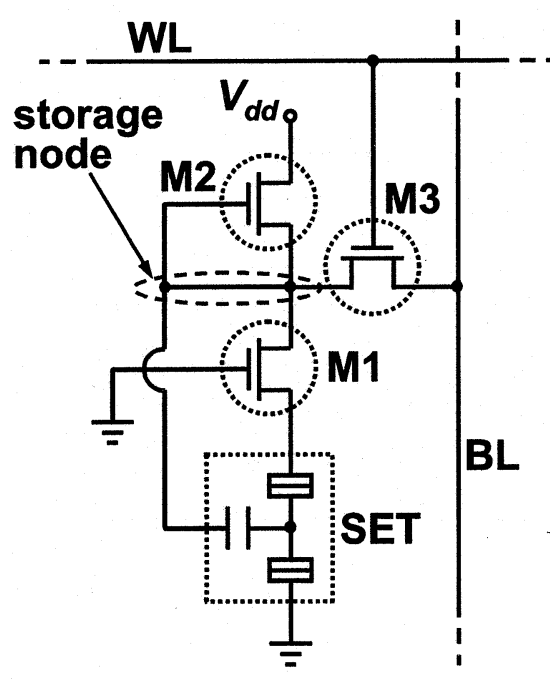

(a)

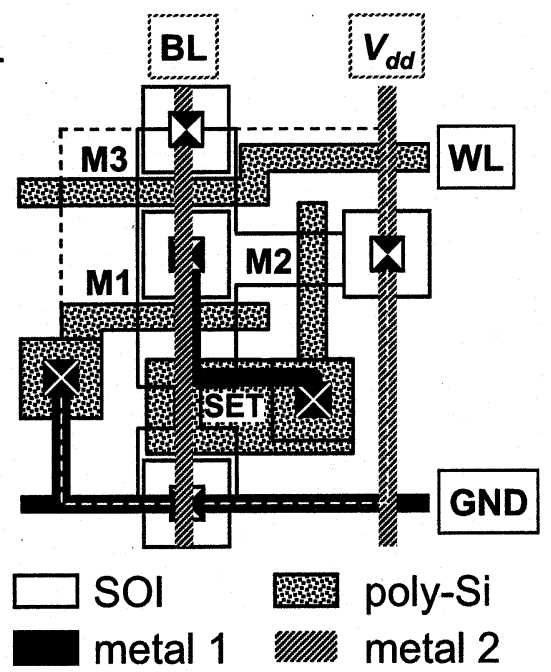

(b)

Fig. 14. Single-electron MV SRAM cell with two depletion-mode MOSFETs (M1 and M2) and one enhancement-mode MOSFET (M3). (b) An example of the SRAM layout. Metal-1 and 2 lines are drawn narrower for clarity. The unit cell area surrounded by the dashed line is $31.1 \boldsymbol{F}^{2}$, where $\boldsymbol{F}$ is half the minimum wiring pitch.

TABLE I

CELl StruCtures to IMPROVE THE OPERATING SPEED OF THE MV SRAM

\begin{tabular}{c|c|c|c}
\hline $\begin{array}{c}\text { storage } \\
\text { node }\end{array}$ & FL \\
\hline $\begin{array}{c}\text { No. of } \\
\text { elements }\end{array}$ & Original & w/ storage capacitor & w/ read-out Trs. \\
\hline $\begin{array}{c}\text { Write-in } \\
\text { speed }\end{array}$ & 4 & 5 & 6 \\
\hline $\begin{array}{c}\text { Read-out } \\
\text { mode }\end{array}$ & Non-destructive & Destructive & Non-destructive \\
\hline $\begin{array}{c}\text { Read-out } \\
\text { speed }\end{array}$ & Slow & Fast & Fast \\
\hline $\begin{array}{c}\text { Comparable } \\
\text { speed to }\end{array}$ & - & Multiple-valued DRAM & Multiple-valued Flash \\
\hline
\end{tabular}

order of magnitude. These changes of parameters would result in a delay time of about 1 ns.

For large-scale memories, different approaches should be taken to improve the speed because the load capacitance, i.e., the BL capacitance, cannot easily be reduced. Other possible cell structures are compared with the original one in Table I. If we add a storage capacitor $\boldsymbol{C}_{\boldsymbol{s}}$ to the storage node (center column of the table), the charges stored in $C_{s}$ can be driven out to the BL in a short time. The small voltage difference on the BL is read by a sense amplifier. The speed should be comparable to that of multiple-valued DRAM [26]. Although the read-out operation is destructive, the data would be refreshed immediately by the sense amplifier. Of course, the SRAM proposed above does not require periodic refreshing. Also note that the area penalty due to the storage capacitor is negligible, since the area above the storage node is open in the proposed cell [Fig. 14(b)].

Speed could also be improved by adding MOSFETs specialized for read-out operation (right column of the table). M4 converts voltages at the storage node to current levels, and M5 controls the access from the sense line (SL). By using a special read-out scheme, such as parallel charge sensing, speed comparable to that of MV flash memory [15], [16] could be attained. Although this cell structure enables nondestructive read-out, cell area is sacrificed considerably due to the increased number of transistors.

\section{Manufacturing Issues}

In the proposed application of a SET to MV logic and memory, control of the oscillation period $\left(e / \boldsymbol{C}_{g}\right)$ and the 
threshold voltage is quite important. If the number of MV levels is $\boldsymbol{N}$, fluctuation of $\boldsymbol{e} / \boldsymbol{C}_{g}$ should be much smaller than the target $\boldsymbol{e} / \boldsymbol{C}_{g}$ divided by $\boldsymbol{N}$, and fluctuation of the threshold voltage should be much smaller than $\boldsymbol{e} / \boldsymbol{C}_{g}$. In the PADOX device, we have found a clear relationship between $\mathrm{Si}$ wire length and $\boldsymbol{C}_{g}$ [27]. This means that size control is critical for attaining an accurate oscillation period. We have also found that the threshold voltage of the SET is related uniquely to $\boldsymbol{C}_{g}$ [28], [31]. This indicates that there is not an apparent effect of random offset charges [29] among the measured devices, and again that size control is crucial for controlling the threshold voltage. Since the densities of interface traps and fixed oxide charges in the $\mathrm{Si}-\mathrm{SiO}_{2}$ system are of the order of $10^{10} \mathrm{~cm}^{-2}$, we cannot ignore the random offset charges in large-scale circuits. Still, the primary concern in the present device is size control. Considering the rapid progress of Si LSI technology [30], we will be able to attain a better control of size and to make SET-MOSFET hybrid circuits with predetermined characteristics in the foreseeable future.

\section{CONCLUSIONS}

We made the best use of the periodic nature of the SET characteristics to construct MV logic and memory. By combining a SET with a MOSFET, which can be fabricated on the same SOI wafer by the PADOX process, we obtained a universal literal gate that has sharp transfer characteristics and large output amplitude. We also verified the operation of a novel quantizer, which is equivalent to a MV static memory, based on the same scheme. These basic components for MV logic and memory are extremely compact in that the literal gate requires only three elements and the quantizer only four. Moreover, the number of periods or quantized levels is infinite in principle and does not affect the circuit size. With these components, we showed that a 3-bit flash ADC and a carry-propagation-free adder for redundant number representation PD2-3 could be made with half the number of elements in a conventional implementation. A MV SRAM cell could be made with an area of $31.1 F^{2}$, which is much smaller than that of a CMOS binary cell. These results open up the possibility of constructing a new class of MV logic and memory with single-electron devices.

\section{ACKNOWLEDGMENT}

The authors thank Dr. Y. Ono for helpful discussions and technical advice in electrical measurements.

\section{REFERENCES}

[1] J. R. Tucker, "Complementary digital logic based on the Coulomb blockade," J. Appl. Phys., vol. 72, pp. 4399-4413, Nov. 1992.

[2] N. Korotkov, R. H. Chen, and K. K. Likharev, "Possible performance of capacitively coupled single-electron transistors in digital circuits," $J$. Appl. Phys., vol. 78, pp. 2520-2530, Aug. 1995.

[3] R. H. Chen, A. N. Korotkov, and K. K. Likharev, "Single-electron transistor logic," Appl. Phys. Lett., vol. 68, pp. 1954-1956, Apr. 1996.

[4] N. J. Stone and H. Ahmed, "Logic circuit elements using single-electron tunneling transistors," Electron. Lett., vol. 35, pp. 1883-1884, Oct. 1999

[5] Y. Ono, Y. Takahashi, K. Yamazaki, M. Nagase, H. Namatsu, K. Kurihara, and K. Murase, "Si complementary single-electron inverter with voltage gain," Appl. Phys. Lett., vol. 76, pp. 3121-3123, May 2000.

[6] G. A. Sai-Halasz, "Performance trends in high-end processors," Proc. IEEE, vol. 83, pp. 20-36, Jan. 1995.
[7] M. T. Bohr, "Interconnect scaling-The real limiter to high performance ULSI," in IEDM Tech. Dig., 1995, pp. 241-244.

[8] S. Kawahito, K. Mizuno, and T. Nakamura, "Multiple-valued current-mode arithmetic circuits based on redundant positive-digit number representations," in Proc. 21st IEEE Int. Symp. on Multiple-Valued Logic, 1991, pp. 330-339.

[9] T. Hanyu, M. Kameyama, and T. Higuchi, "Beyond-binary circuits for signal processing," in Dig. Tech. Papers, IEEE Int. Solid-State Circuit Conf., 1993, TA 8.6, pp. 134-135.

[10] L. J. Micheel, "Heterojunction bipolar technology for emitter-coupled multiple-valued logic in gigahertz adders and multipliers," in Proc. 22nd IEEE Int. Symp. on Multiple-Valued Logic, 1992, pp. 19-26.

[11] H. C. Lin, "Resonant tunneling diodes for multi-valued digital applications," in Proc. 24th IEEE Int. Symp. on Multiple-Valued Logic, 1994, pp. $188-195$.

[12] D. Etiemble, "On the performance of multivalued integrated circuits: Past, present and future," in Proc. 22nd IEEE Int. Symp. on MultipleValued Logic, 1992, pp. 156-164.

[13] M. Akazawa, K. Kanaami, T. Yamada, and Y. Amemiya, "Multiplevalued inverter using a single-electron-tunneling circuit," IEICE Trans. Electron, vol. E82-C, pp. 1607-1614, 1999.

[14] Y. Suda, N. Shiotani, K. Imure, K. Hirohashi, and K. Yamamura, "Single-electron multiple-valued base-n full adder circuit using a bunch of electrons as a unit logic value," in Abs. 2001 Silicon Nanoelectronics Workshop, Kyoto, June 2001, pp. 32-33.

[15] M. Bauer et al., "A multilevel-cell $32 \mathrm{Mb}$ flash memory," in Dig. of Tech. Papers. 41st ISSCC, 1995, pp. 132-133.

[16] A. Fazio and M. Bauer, "Intel StrataFlash memory technology development and implementation," Intel Technol. J., 1997. [Online]. Q4'97 Available: http://www.intel.com/technology/itj/q41997/pdf/development.pdf.

[17] L. Guo, E. Leobandung, and S. Y. Chou, "A silicon single-electron transistor memory operating at room temperature," Science, vol. 275, pp. 649-651, Jan. 1997.

[18] H. Sunamura, H. Kawaura, T. Sakamoto, and T. Baba, "Multiple-valued memory operation using a single-electron device: A proposal and an experimental demonstration of a ten-valued operation,"Jpn. J. Appl. Phys., vol. 41, pp. L93-L95, Feb. 2002.

[19] H. Inokawa, A. Fujiwara, and Y. Takahashi, "Multipeak negative-differential-resistance device by combining single-electron and metal-oxide-semiconductor transistors," Appl. Phys. Lett., vol. 79, p. 3620, Nov. 2001

[20] Y. Takahashi et al., "Size dependence of the characteristics of Si singleelectron transistors on SIMOX substrates," IEEE Trans. Electron Devices, vol. 43, pp. 1213-1217, Aug. 1996.

[21] S. Horiguchi et al., "Mechanism of potential profile formation in silicon single-electron transistors fabricated using pattern-dependent oxidation,” Jpn. J. Appl. Phys., vol. 40, pp. L29-L32, Jan. 2001.

[22] "The subthreshold slopes and the $V_{t h}$ shift are large compared to the voltage applicable to the SET $(28 \mathrm{mV})$. The subthreshold slopes are also large for the low measurement temperature $(27 \mathrm{~K})$ and the small thichness ratio of the gate oxide and the burried oxide $(90 \mathrm{~nm} / 400 \mathrm{~nm})$. Superposition of the characteristics for area and edge channels is probably the cause of the large subthreshold slopes.,"

[23] P. R. Gray and R. G. Meyer, Analysis and Design of Analog Integrated Circuits, 3rd ed. New York: Wiley, 1993, pp. 225-227, 464-465.

[24] S. J. Ahn and D. M. Kim, "Asynchronous analogue-to-digital converter for single-electron circuits," Electron. Lett., vol. 34, pp. 172-173, January 1998.

[25] Y. Mizugaki and P. Delsing, "Single-electron signal modulator designed for a flash analog-to-digital converter," Jpn. J. Appl. Phys., pt. 1, vol. 40, no. 10, pp. 6157-6162, Oct. 2001.

[26] T. Okuda and T. Murotani, "A four-level storage 4-Gb DRAM," IEEE J. Solid-State Circuit, vol. 32, pp. 1743-1747, Nov. 1997.

[27] M. Nagase, S. Horiguchi, A. Fujiwara, and Y. Takahashi, "Three dimensional size evaluation of island in Si single-electron transistor," in Ext. Abst. Conf. Solid State Devices Mater., Tokyo, Japan, 2001, pp. 436-437.

[28] A. Fujiwara, S. Horiguchi, M. Nagase, and Y. Takahashi, "Threshold voltage of Si single-electron transistor," in Ext. Abst. Conf. Solid State Devices Mater, Nagoya, Japan, 2002, pp. 110-111.

[29] K. K. Likharev, "Single-electron devices and their applications," Proc. IEEE, vol. 87, pp. 606-632, Apr. 1999.

[30] The international technology roadmap for semiconductors. Semicond. Ind. Assoc. [Online]. Available: http://public.itrs.net/Files/2001ITRS/ Home.htm.

[31] A. Fujiwara, S. Horiguchi, M. Nagase, and Y. Takahashi, "Threshold voltage of Si single-electron transistor," Jpn. J. Appl. Phys., submitted for publication. 


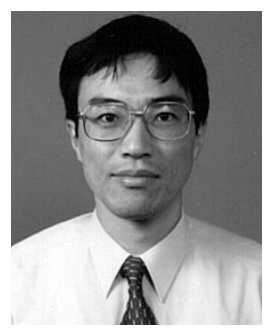

Hiroshi Inokawa (M'90) received the B.S., M.S., and $\mathrm{Ph} . \mathrm{D}$. degrees in electrical engineering from Kyoto University, Kyoto, Japan, in 1980, 1982, and 1985 , respectively.

In 1985, he joined the Atsugi Electrical Communications Laboratories, Nippon Telegraph and Telephone Corporation (NTT), Kanagawa, Japan, where he was engaged in research and development of scaled-down MOS devices including those with reduced parasitic effects, and fully-depleted bulk MOSFETs. From 1989 to 1990, he was a Visiting Researcher at Microelectronics Laboratory, Santa Clara University, CA, where he researched the degradation and recovery of electrically stressed MOS devices. From 1990 to 1997, he focused on the process integration of $0.2-\mu \mathrm{m}$ bulk CMOS, especially for advanced isolation schemes with deep and shallow trenches. From 1997 to 2000, he was engaged in technology transfer and production management of $0.25-\mu \mathrm{m}$ bulk and SOI CMOS LSIs as the Engineering Director in LSI Production Division of NTT Electronics Corporation, Kanagawa. Currently he is concentrating on silicon nanodevices, particularly single-electron devices, and their applications. He is now a Senior Research Engineer, Supervisor at NTT Basic Research Laboratories, Kanagawa.

Dr. Inokawa is a member of the Institute of Electronics, Information, and Communication Engineers of Japan and the Japan Society of Applied Physics.

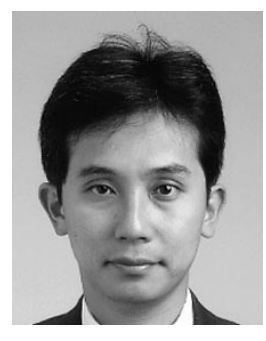

Applied Physics.
Akira Fujiwara (M'97) was born in Japan in 1967. He received the B.S., M.S., and Ph.D. degrees in applied physics from the University of Tokyo, Japan, in 1989, 1991, and 1994, respectively.

In 1994, he joined LSI Laboratories, Nippon Telegraph and Telephone (NTT) Corporation, Kanagawa, Japan. In 1996, he moved to the NTT Basic Research Laboratories. Since 1994, he has been engaged in research on the physics of silicon nanostructures and their application to single-electron devices.

Dr. Fujiwara is a member of the Japan Society of

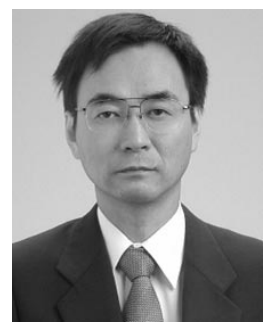

Yasuo Takahashi (M'95) received the B.S., M.S., and $\mathrm{Ph} . \mathrm{D}$. degrees in electronics from Tohoku University, Sendai, Japan, in 1977, 1979, and 1982, respectively.

In 1982, he joined the Musashino Electrical Communication Laboratories, Nippon Telegraph and Telephone Public Corporation (NTT), Tokyo, Japan. Since then, he has been engaged in research on the physics and chemistry of the surface and interface of semiconductors. His current work includes research on the quantum physics of Si nano-structures and its electronic device applications particularly to Si single-electron devices. Since 1996, he has been with NTT Basic Research Laboratories, Kanagawa, Japan. Currently, he is the Leader of the Silicon Nanodevice Research Group and the Executive Manager of the Device Physics Laboratory.

Dr. Takahashi is a member of the Japan Society of Applied Physics. 